\title{
Sistema de informação para gestão de custos operacionais
}

\begin{abstract}
Márcio Roberto de Mello Mestrado em Ciências Contábeis pela Universidade do Vale do Rio dos Sinos UNISINOS
\end{abstract} Av. Unisinos, 950. Bairro Cristo Rei. São Leopoldo/RS. CEP: 93.022-000 Email:mello@mellomiotto.com.br

João Luiz Becker PhD em Management Science - UCLA/USA Professor da Universidade Federal do Rio Grande do Sul - UFRGS Rua Washington Luís, 855. Centro. Porto Alegre/RS. CEP: 90010-460

Email: jlbecker@ea.ufrgs.br

Daniel Porto Engenheiro Eletricista pela Pontifícia Universidade Católica do Rio Grande do Sul PUCRS

AES Sul Distribuidora Gaúcha de Energia Presidente Roosevelt, 68. São Leopoldo/RS. CEP: 93010-060 E-mail: daniel.porto@aes.com

\author{
Adolfo Alberto Vanti \\ Doutorado em Direção de empresas - DEUSTO/ESPANHA \\ Professor da Universidade do Vale do Rio dos Sinos - UNISINOS \\ Av. Unisinos, 950. São Leopoldo/RS. CEP: 93.022-000 \\ Email: avanti@unisinos.br
}

\section{RESUMO}

Este trabalho com ênfase em custos é resultado de uma pesquisa aplicada no setor elétrico. Foram pesquisados os temas: Sistema de Informação, Sistema de Custos e Metodologia Design Research para geração de artefato computacional. O problema de pesquisa está relacionado com o desenvolvimento de sistema de informação para gestão dos custos operacionais e o resultado do estudo aponta uma melhoria dos critérios de cálculo da estrutura de custos. Este sistema permite simular cenários de remuneração de atividades em energia elétrica.

Palavras-chave: Custos. Design research. Sistema de apoio à decisão. 
Sistema de informação para gestão de custos operacionais Márcio Roberto de Mello, João Luiz Becker, Daniel Porto, Adolfo Alberto Vanti

\section{ABSTRACT}

This work with emphasis on costs management is a result of applied research in the electricity sector. We examined the issues of Information Systems, Cost System and Design Research Methodology for the generation of computational artifact. The research problem is related with the development of information system for of operational costs management. The final scope of this type of work is an improvement of the criteria for calculating the cost structure. This system allows simulating scenarios remuneration in electricity activities.

Key-Words: Costs. Design research. Decision support system.

\section{INTRODUÇÃO}

Recentemente o setor de energia elétrica passou por um período de reestruturação com privatizações e consolidação de modelo de gestão. As tarifas energéticas junto ao "cliente cativo" (não escolhe o fornecedor) são revisadas continuamente com vistas a proporcionar um preço ainda mais justo ao cidadão.

O setor energético exige então metodologias e instrumentos cada vez mais eficazes para o gerenciamento de custos. Nesse sentido, Bornia e Dutra (2009) salientam a questão da necessidade das distribuidoras de energia elétrica maximizar seus resultados nesse novo mercado ainda mais competitivo.

Uma parceria universidade-empresa ensejou a realização de um projeto relacionado à análise e desenvolvimento de um modelo para custeio de serviços de equipes de manutenção de rede aérea. Essa parceria foi regulada pela Aneel, seguindo suas diretrizes de P\&D com a participação de pesquisadores com diferentes titulações. No trabalho foram contemplados conceitos de estatística, custos e sistema de informação de maneira a implementar um modelo para gerenciar a remuneração das equipes terceirizadas $\mathrm{H}$ e linha Viva.

A equipe de trabalho desenvolveu uma estratégia de avanço no projeto baseada na seguinte questão de pesquisa: Como simular custos a partir das atualizações da estrutura de custos e dos tempos e movimentos das atividades realizadas na construção de redes de distribuição de energia elétrica? Assim, este etudo teve como objetivo desenvolver um sistema de informação para apoiar as decisões de 
remuneração, quando das atualizações da estrutura de custos e dos tempos e movimentos das atividades realizadas na construção de redes de distribuição.

Buscou-se atingir o objetivo geral do trabalho através de dois objetivos específicos, assim definidos: aferir os custos, tempos e movimentos atualmente praticados por estas equipes e; garantir que o sistema permita a simulação de modificações na estrutura de custos das equipes e dos tempos e movimentos das atividades realizadas por elas.

Estudos como este contribuem no sentido de promover uma análise crítica da gestão de remuneração sobre processos terceirizados. Estes são fundamentais, uma vez que estas empresas realizam investimentos previamente acordados com 0 regulador, e que grande parte destes investimentos está relacionada com a construção e manutenção de seus ativos.

$\mathrm{Na}$ continuação são apresentados conceitos de sistemas de informação com característica de apoio à decisão, sistema de custos e metodologia; conclusão e referências bibliográficas.

\section{FUNDAMENTAÇÃO TEÓRICA}

\subsection{Sistema de Apoio à Decisão (SAD)}

Sistema de informação (SI) é um sistema que coleta, processa, armazena, analisa e dissemina informações para um propósito específico (TURBAN et al., 2010). Este tipo de sistema gera um programa aplicativo que apoia a realização de um processo de negócio como o de mensuração de custos, gerando assim informação gerencial e conhecimento que posteriormente é aplicado em forma de alguma inovação.

Um programa aplicativo na tomada de decisão se consolidou com a denominação Sistema de Apoio à Decisão ou Decision Support System (DSS), pois este tipo de sistema contribui para a resolução de problemas pouco ou não estruturados (KEEN; SCOTT-MORTON, 1978; SPRAGUE; CARLSON, 1982; SPRAGUE; WATSON, 1989). 
Sistema de Apoio à Decisão (SAD) é um tipo de sistema que evoluiu por diferentes etapas desde $\mathrm{O}$ atendimento ao propósito de armazenar informação necessária para a tomada de decisão até o momento atual que incorpora modelos matemáticos robustos como os baseados em lógica difusa. Assim, esse tipo de sistema não só provê o gestor de informações, mas também analisa diferentes alternativas considerando associações e tendências na garimpagem de dados (Data Mining), estas geradas a partir de grandes depósitos de dados (Data Warehouse) (WITTEN; FRANK, 2005).

Neste trabalho, a parte de maior robustez matemática que caracteriza o sistema final está relacionada à avaliação de atividades terceirizadas executadas (transportar postes, aterrar, isolar, trocar portes e etc), envolvendo análises estatísticas e probabilidades calculadas, bem como na composição e simulação do sistema de custos. Devido a este enfoque de custos, na sequência a ênfase da fundamentação está relacionada com o Sistema de Custos.

\subsection{Sistema de Custos}

Pode-se entender o conceito de sistema de custos como o conjunto de métodos e técnicas que uma empresa utiliza para coletar e organizar os dados físicos e monetários do processo operacional e transformá-los em informações para avaliação de estoques e determinação de lucros, bem como para apoiar o processo decisório e o planejamento (BACKER; JACOBSEN, 1977).

Um sistema de custos é composto por três componentes principais, como revela a Figura 1: os métodos de acumulação de custos, os métodos de custeio e o método de controle dos custos.

O sistema de acumulação de custos preocupa-se com a coleta e acumulação dos componentes físicos do sistema de custos (tempos e materiais consumidos ao longo de um processo). O sistema de custeio preocupa-se com a valorização desses fatores (custos das horas trabalhadas e dos materiais consumidos). Trata-se, nesse caso, dos componentes monetários do sistema. 
Por fim, tem-se o método de controle de custos. O custo real é o efetivamente ocorrido, também denominado de realizado, que deve ser apurado todos os meses a partir do encerramento contábil do período. O custo-padrão é o custo gerencial, projetado, pré-determinado, conforme abordado com detalhes no desenvolvimento do trabalho. Na Figura 1 é possível visualizar o sistema de custos.

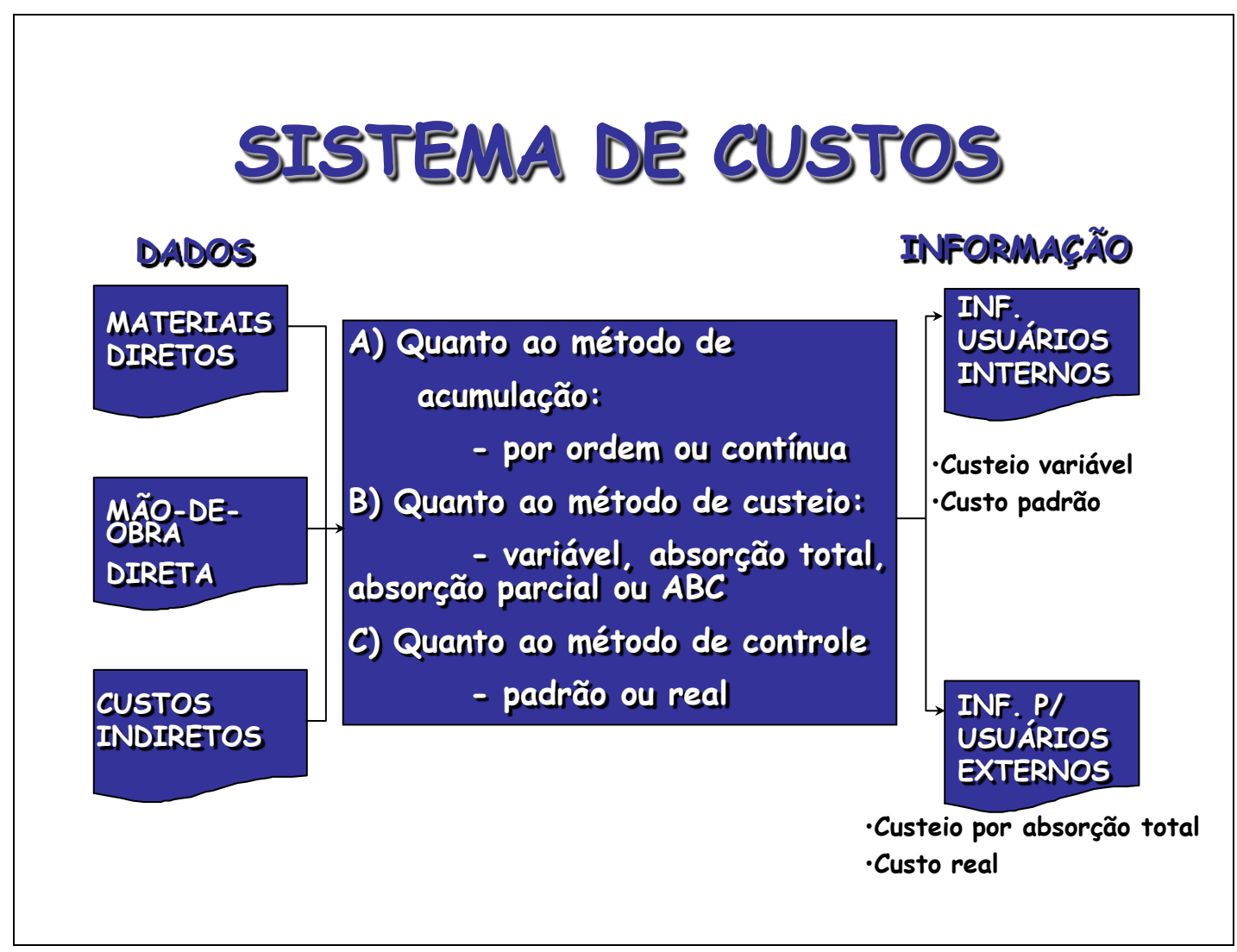

Figura 1: Sistema de Custos

Fonte: Mello (2002).

Essa visão sistêmica e conceitual torna-se relevante a partir do momento em que se compreende que, conforme o método de custeio utilizado por uma empresa tem-se uma informação de custo de produto ou serviço diferente em termos de valores monetários. Pode-se considerar que o grande potencial informativo de um sistema de custos depende da adequada escolha e combinação de seus diversos componentes, 
considerando o tipo de informação que se deseja e que decisões são tomadas a partir das mesmas.

A seguir são revisados os principais conceitos relacionados aos componentes anteriormente citados.

\subsubsection{Sistemas de Acumulação de Custos}

Existem dois fatores que determinam o tipo de custeio, se por processo contínuo ou por ordem e a adequação contábil administrativa. Quanto à forma, se a empresa trabalha produzindo produtos iguais de forma contínua (um ou vários) e fundamentalmente para estoque, isto é, para venda, já terá caracterizada sua natureza. Produz-se atendendo a encomendas dos clientes ou, então, produz-se também para venda posterior, mas de acordo com determinações internas especiais, não de forma contínua, mas sim entre as de produção por ordem (MELLO, 2002).

\subsubsection{Métodos de Custeio}

Custeio significa método de apropriação de custos, sendo que são vários os sistemas existentes e utilizados, como o Custeio por Absorção, o Custeio Variável e o Custeio Baseado em Atividades (SLOMSKI, 2006).

\subsubsection{Custeio por absorção total (integral)}

Conforme Martins (1996, p. 41), este método de custeio "consiste na apropriação de todos os custos de produção aos bens elaborados, e só os de produção; todos os gastos relativos ao esforço de fabricação são distribuídos para todos os produtos processados".

Bornia (2010) segue na mesma linha conceitual ao afirmar que o custeio por absorção total consiste na apropriação de todos os custos incorridos no processo de fabricação de todos os produtos da empresa, quer estejam diretamente vinculados ao produto, quer se refiram à tarefa de produção em geral, sendo neste caso alocados aos bens fabricados, mediante rateio. Serve também para a avaliação de estoques. 
Ao realizar o rateio de custos e despesas fixas aos mesmos, a empresa está utilizando algum critério de rateio, ou seja, está definindo alguma base de rateio. Estes critérios e/ou bases, podem ser muito subjetivos uma vez que são definidas e estipuladas por alguém que aplicou o seu julgamento pessoal para esta determinação. Dessa forma, conforme se modifica o critério anteriormente determinado, modifica-se todo o resultado apresentado, tanto em relação ao custo do produto ou serviço como em relação à avaliação de resultado da empresa (MELLO, 2002).

Um segundo motivo para o seu não uso gerencial é o fato de que esse sistema considera como base para a apropriação dos custos o volume de produtos ou serviços realizados no período, independente da capacidade instalada da empresa. Isso significa que a ociosidade/ineficiência da empresa é repassada aos produtos ou serviços, ou seja, é repassada ao mercado.

\subsubsection{Custeio por absorção parcial (ideal)}

Semelhantemente ao custeio por absorção total, o custeio por absorção parcial também tem como premissa básica apropriar todos os custos, tanto fixos como variáveis, aos produtos ou serviços que uma empresa executa. A significativa diferença é que enquanto o primeiro realiza essa apropriação com base no volume de negócios realizados no mês, o segundo considera como base de apropriação a capacidade física instalada da empresa. Conforme Souza e Diehl (2009, p.104) no tipo absorção ideal "os produtos somente consomem uma parcela eficiente dos recursos, sendo os demais perdidos por ineficiência e ociosidade".

$\mathrm{Na}$ visão de Bornia (2010), neste método consideram-se todos os custos como sendo custo do produto, porém o custo relacionado com os insumos usados de forma não-eficiente não é apropriado ao produto. O método se adapta ao auxílio do controle de custos e ao apoio ao processo de melhoria contínua da empresa, já que possibilita a ação ao trabalho que não agrega valor e as perdas propriamente ditas.

A utilização da capacidade instalada como base de apropriação de custos, faz com que os custos relativos à ineficiência/ociosidade não sejam repassados aos produtos e serviços produzidos no período. Garante ainda a apropriação de todos os 
custos fixos e variáveis, permitindo que a empresa busque a otimização da sua estrutura e não transfira sua ineficiência ao mercado.

\subsubsection{Custeio variável}

Como se identificou ao analisar tanto o custeio por absorção total como o custeio por absorção ideal, ambos apresentam questionamentos em função da utilização de técnicas de rateio para alocar custos fixos aos produtos/serviços processados. Em função destas dificuldades e de distorções de avaliação, surgiu o custeio variável como uma alternativa de custeamento.

Com base nesse método, somente são alocados aos produtos e serviços os custos variáveis, ficando os custos fixos separados e considerados como despesas do período, indo diretamente para o resultado. Um conceito muito importante é o da margem de contribuição, que é a diferença entre as receitas e os custos e despesas variáveis, significando a sobra gerada pelas vendas suficiente para cobrir os custos e despesas fixas e formar o resultado da empresa.

Na visão de Padoveze (2003), o custeio variável tem como desvantagens o entendimento que os custos variáveis e os custos fixos são necessários para a elaboração dos produtos e a estes devem ser atribuídos. Não segue o princípio contábil da confrontação das receitas com os custos e despesas e pode prejudicar a análise por parte de credores (índices de liquidez e capital circulante líquido). Não considera os custos fixos na determinação do preço de venda, os custos fixos não são completamente fixos e os variáveis não são completamente variáveis.

Podem-se citar muitas vantagens na utilização do custeio variável, dentre as quais se destacam: o impacto dos custos fixos no resultado é salientado, uma vez que o total destes custos aparece na demonstração do resultado; as informações para análise das relações custo $x$ volume $x$ lucro são obtidas de forma mais fácil do que no sistema por absorção; adapta-se melhor aos instrumentos de controle da organização, tais como custo-padrão e orçamento; o lucro de um período não é afetado pelas flutuações causadas pela absorção, maior ou menor, dos custos fixos aos produtos. 


\subsubsection{Custeio baseado em atividades}

Segundo Atkinson et al. (2000), o Custeio Baseado em Atividades (ABC) parte da premissa que os produtos consomem atividades e que estas atividades é que consomem os recursos, ao contrário dos demais métodos de custeio, nos quais os produtos consomem diretamente os recursos. Surgiu com o objetivo de permitir uma adequada apropriação dos custos e despesas fixas aos objetos de custos de uma empresa.

Ao contrário dos métodos de custeio tradicionais (absorção total e absorção parcial) não utiliza rateios com base em volume físico de produtos ou em valor de receita de produtos. Trabalha com o conceito do direcionador de custo, que indica o quanto de atividades os produtos estão consumindo, independentemente dos respectivos volumes.

Para Nakagawa (1995), representa uma transação que determina a quantidade de trabalho (não a duração) e, por meio dela, o custo de uma atividade. Também pode ser entendido como um fator causal que influencia o nível e o desempenho de atividades e o consumo resultante de recursos.

Após considerar as características dos quatro métodos de custeio existentes, detalha-se o último componente do sistema de custos, que é o método de controle de custos.

\subsubsection{Métodos de Controle de Custos}

O Custo Real é o custo incorrido no período, apurado a partir das informações contábeis, enquanto que o Custo-Padrão é o custo projetado e calculado a partir do que deverá ocorrer (LEONE, 2000), estabelecido pela adoção de métodos racionais que utilizam a projeção e a experimentação.

\subsubsection{Custo real}

O custo real, nada mais é do que o custo apurado do produto ou serviço de cada período, com base nas informações contábeis elaboradas. Para a apuração do custo real dos produtos/serviços mensais, os gastos são apropriados aos mesmos tomando 
como base a real e efetiva utilização da capacidade de produção. Dessa forma, o custo real absorve toda a ineficiência/ociosidade da operação no respectivo mês.

Como instrumento de planejamento estratégico ou operacional, o custo real não tem nenhum significado, pois representa o custo acontecido, sendo nesses casos substituído pelo custo-padrão. O custo real tem validade apenas para analisar as causas dos desvios, das variações. Para o dia-a-dia o custo-padrão representa muito mais utilidade (MELLO, 2002).

\subsubsection{Custo-padrão}

Um padrão é uma referência (benchmark) ou norma para a avaliação do desempenho. Padrões são amplamente empregados na contabilidade gerencial, estando relacionados à quantidade e ao custo dos insumos empregados na produção dos bens ou na prestação dos serviços. Para Martins (2008), a forma mais eficaz de se controlar custos é a partir da institucionalização do custo-padrão, utilizável tanto no sistema de custeio por absorção como no sistema de custeio variável.

Ainda o mesmo autor apresenta diversas acepções de custo-padrão. Muitas vezes, o custo-padrão é compreendido como se fosse o custo ideal de fabricação de determinado produto ou serviço. Nestas condições, seria o valor obtido a partir da utilização das melhores matérias-primas possíveis, da mais eficiente mão-de-obra, da utilização de $100 \%$ da capacidade instalada da empresa.

Em contrapartida, existe outro conceito denominado custo-padrão corrente. Este considera as deficiências que normalmente existem e ocorrem nas empresas em termos de equipamentos, qualidade de materiais, mão-de-obra, entre outros. É um valor que a empresa considera difícil de alcançar, mas perfeitamente possível.

Como terceira possibilidade, há o custo estimado. Este parte da hipótese de que a média de um período passado é um número válido e apenas introduz algumas modificações esperadas, tais como mudança de equipamentos, volume de atividades, etc. 
O custo-padrão corrente efetiva a união entre aspectos teóricos e práticos, considerado assim mais científico, considerando aspectos práticos e não apontando defeitos ou ineficiências.

Após a abordagem dos principais conceitos que fundamentaram este trabalho, contextualiza-se a Metodologia Design Research (DR) utilizada nesta pesquisa para, finalmente, apresentar o Modelo desenvolvido.

\section{METODOLOGIA}

A abordagem adotada na pesquisa foi a design science, a qual objetiva desenvolver conhecimento que possa ser usado por profissionais em seus campos de atuação para resolução de problemas (AKEN, 2005). Sua implementação se converte em Design Research (DR), uma técnica, conforme menciona Manson (2006), que envolve uma análise de uso e de desempenho de um ou mais artefatos, que serve para compreender, explicar e melhorar o comportamento de uma realidade organizacional. É uma metodologia que permite a aplicação de técnicas qualitativas e quantitativas, assim como permite uma análise e interpretação dos dados sob qualquer uma das epistemologias (GRILO, 2008).

Na generalidade das aplicações de Design Research são criados quatro tipos de informações (MARCH; SMITH 1995): Construtores, Modelos, Métodos e Instâncias. Os construtores correspondem a vocabulário que constitui a definição do problema ou domínio da solução. Os modelos são conjuntos de pressupostos ou declarações que expressam os relacionamentos entre os construtores identificados. Métodos são constituídos por conjuntos de passos que são necessários para completar uma determinada tarefa. Finalmente, instâncias correspondem à implementação do artefato num ambiente, ou seja, a aplicação dos construtores, modelos e métodos. Então, considerando essa metodologia ilustrada a seguir, a questão-problema foi tratada com ênfase em três aspectos:

- A revisão dos tempos de realização das atividades;

- A estrutura de custos a ser adotada; e 
Sistema de informação para gestão de custos operacionais Márcio Roberto de Mello, João Luiz Becker, Daniel Porto, Adolfo Alberto Vanti

- A composição de um sistema de apoio à decisão.

Estes três aspectos seguiram por um fluxo de DR conforme Figura 2 da metodologia realizada.

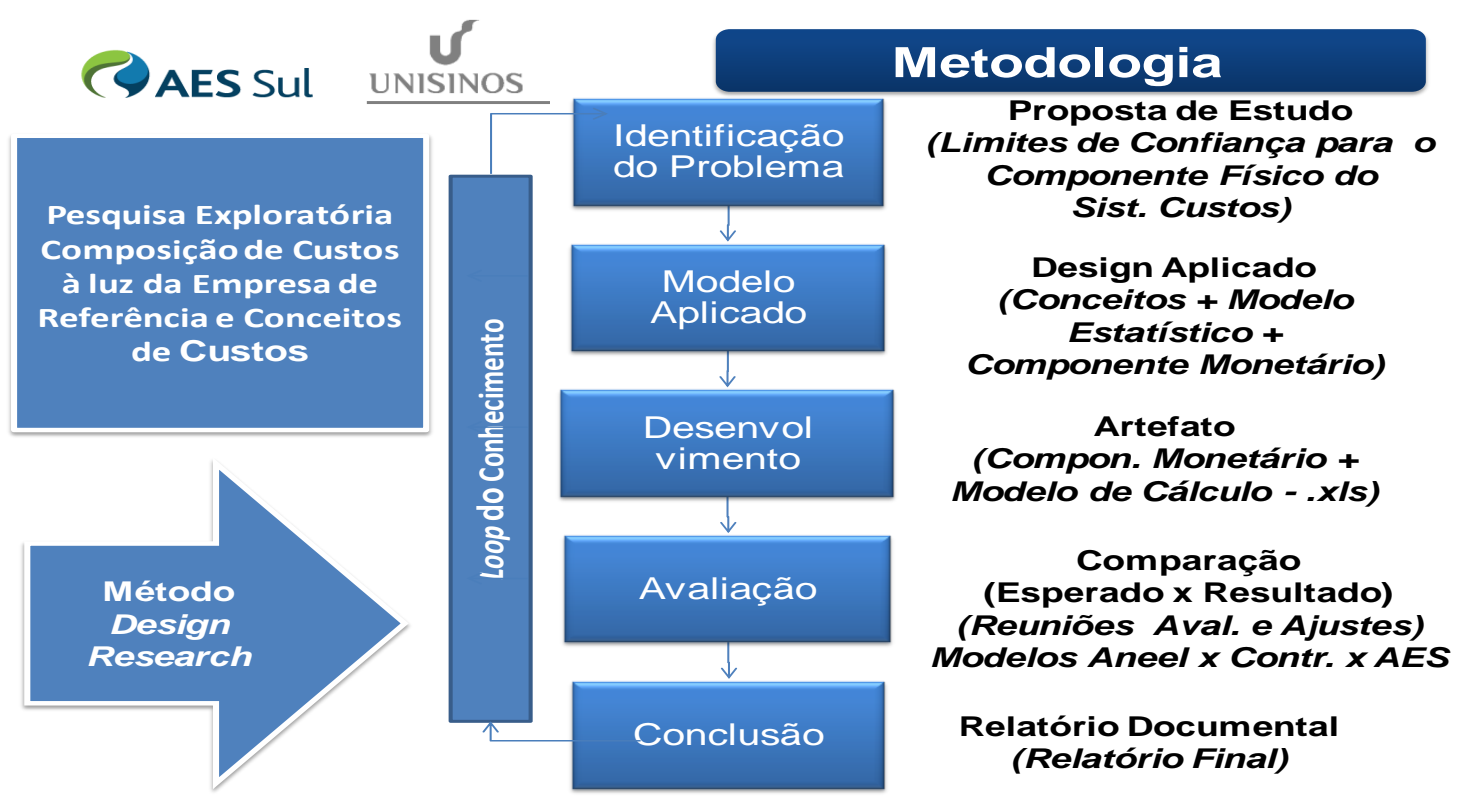

Figura 2: Metodologia utilizada

Fonte: os autores.

Com base no conhecimento dos participantes da pesquisa e no embasamento teórico, foi estruturado, testado e validado o Design. As etapas foram executadas de forma interativa durante a pesquisa, uma vez estabelecida a proposta inicial e novas informações que foram sendo obtidas.

\section{CONTEXTO DE APLICAÇÃO DA PESQUISA}

A empresa estudada AES SUL atua na região centro-oeste do estado do Rio Grande do Sul, atendendo a 118 municípios. Para o estudo em questão, o sistema proposto foi direcionado para as equipes pesadas de construção de redes de distribuição, que possuem alto grau de terceirização, chegando a representar $85 \%$ do total de atividades realizadas. As etapas também consideram: 
Sistema de informação para gestão de custos operacionais Márcio Roberto de Mello, João Luiz Becker, Daniel Porto, Adolfo Alberto Vanti

\subsection{Revisão dos tempos de realização das atividades}

Nesta etapa foi contemplado o Componente Físico do Modelo que considerou o Conhecimento do negócio (empresa estudada), o Levantamento de Campo com base no modelo de coleta de dados e finalmente e o Desenvolvimento de Modelo Estatístico para validação da amostra.

Contemplaram-se testes de hipóteses para suposição de normalidade, testes KS e no seu final foram gerados os Limites de Confiança nos tempos para alimentar o sistema de custos, sendo processados em SPSS e convertidos em Excel, bem como também utilizado o software Sphinx para tratamento de atividades não remuneradas como o check list de segurança. Na figura 3 é apresentado o fluxo de tratamento estatístico.

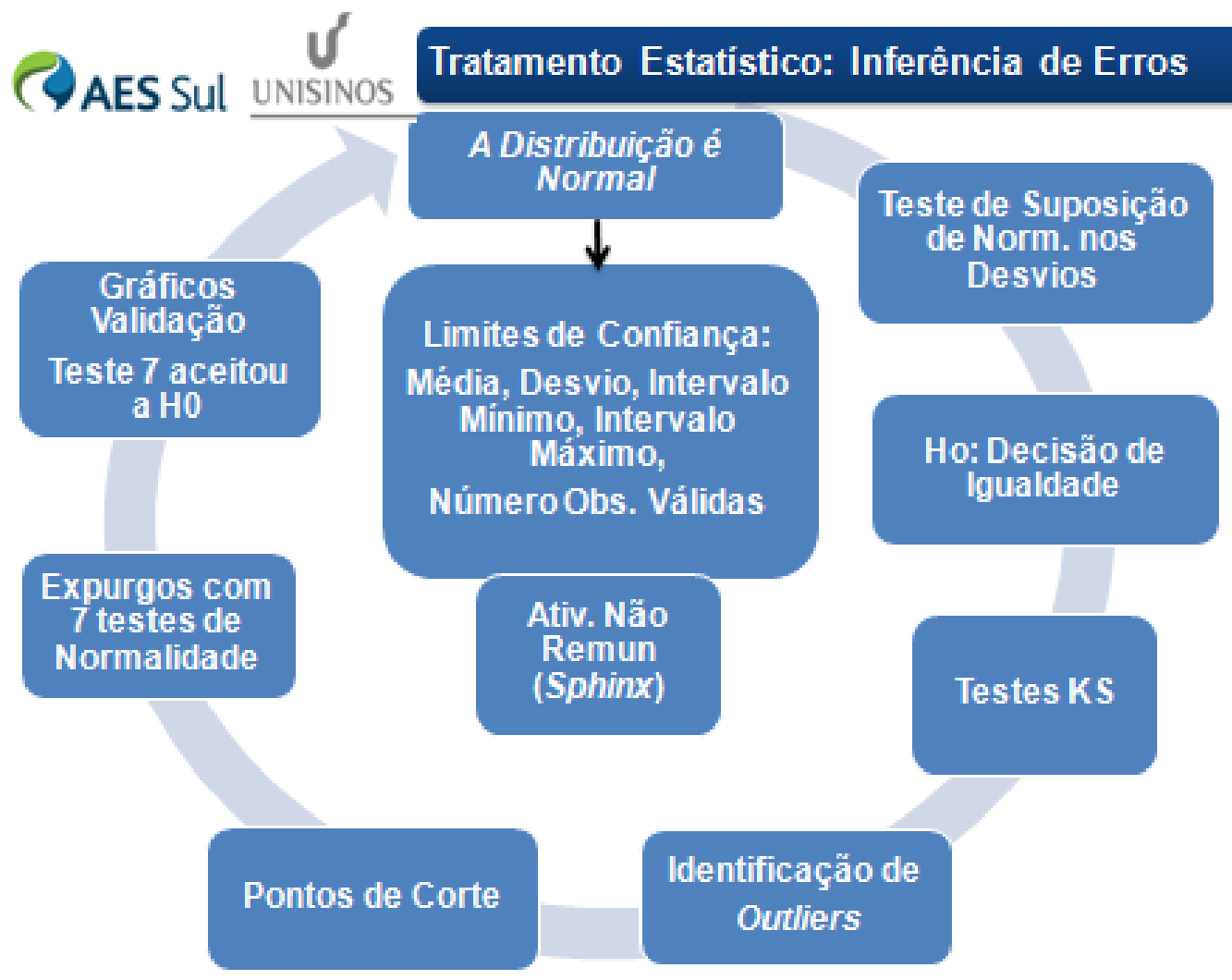

Figura 3: Tratamento Estatístico

Fonte: os autores. 
O processo final de análise estatística também foi precedido por etapas relacionadas: coleta de dados realizada por empresa contratada, tabulação de dados, acumulação de dados, cálculo de médias e desvios, transposição de dados para a análise estatística, usando o software estatístico SPSS. Com isso, realizou-se a etapa anterior representada graficamente.

\subsection{Estrutura de custos adotada}

Para a etapa de estruturação de custos adotada considerou-se o Modelo de Custeio apoiado por sistemas de informação e o próprio conceito de DR contextualizado como uma metodologia da Design Science (DS). Este componente envolveu:

- Alinhamento conceitual: validação do modelo de custeio;

- Estudo do modelo ANEEL (empresa de referência ER) e dos materiais de apoio fornecidos pela empresa estudada;

- Detalhamento da própria estrutura de custos do serviço prestado;

- Construção do Modelo de Custos da empresa estudada;

- Desenvolvimento da metodologia em Excel, permitindo a atualização das variáveis e respectivas simulações, que posteriormente será integrado a sistema corporativo da empresa estudada;

- Desenvolvimento do Artefato: considerou o componente Monetário acrescido do modelo de Cálculo, convertido posteriormente em formato .xlsx;

- Avaliação: foi realizada a comparação entre o Planejado e o Realizado alcançado através de diversas reuniões com responsáveis das áreas. Com isso ajustes foram sendo realizados que contemplaram ainda mais os conceitos de sistema de apoio à decisão, ou seja, processo interativo de resolução de problemas pouco ou não estruturados;

- Modelos ANEEL x Contratada x Empresa estudada: foi realizado um comparativo através de cenários e ponto de equilíbrio para analisar conjuntamente a performance do modelo de referência da ANEEL (ER) com empresa contratada modelo e a empresa estudada; 
- Conclusão: O Relatório Documental foi segregado em relatórios trimestrais e ao seu final foi gerado um relatório executivo final. $O$ relatório envolvendo a parte estatística gerou mais de dois milhares de páginas devido aos testes de hipóteses na busca da curva normal, gaussiana;

\subsection{Composição do sistema desenvolvido}

A composição do sistema desenvolvido SAD seguiu a estrutura representada na figura a seguir, elaborada a partir do modelo desenvolvido em formato xlsx. Cabe destacar que os componentes de custos do modelo têm seus respectivos detalhamentos em outras planilhas de apoio vinculadas, que podem ser alteradas, caracterizando a funcionalidade do simulador, que é o objetivo final.

Pode-se simular a estrutura das equipes em termos de quantidade de pessoas, os equipamentos e ferramentas utilizados, o período de depreciação, o valor de custo de aquisição, a quantidade de horas que as equipes trabalharão (capacidade instalada versus utilização efetiva); a quantidade de horas extras, o nível de eficiência aceitável, as taxas de remuneração dos investimentos e de lucro das contratadas, os impostos sobre a receita, o cálculo do ponto de equilíbrio das contratadas, entre outras simulações. 
Sistema de informação para gestão de custos operacionais Márcio Roberto de Mello, João Luiz Becker, Daniel Porto, Adolfo Alberto Vanti

\begin{tabular}{|c|c|c|c|c|c|c|}
\hline \multicolumn{2}{|c|}{ COMPOSIÇÃO DO CUSTO PADRÃO DE UMA EQUIPE H } & $\begin{array}{c}\text { TURMA DE } \\
\text { APOIO }\end{array}$ & $\begin{array}{c}\text { TURMA DE } \\
\text { CONSTRUÇÃOO }\end{array}$ & $\begin{array}{l}\text { CUSTO TOTAL } \\
\text { DA EQUIPE }\end{array}$ & $\begin{array}{l}\text { ADMINISTRAÇÃO } \\
\text { INDIRETA }\end{array}$ & TOTAL GERAL \\
\hline A - CUSTOS COM PESSOAL & BASE PESSOAL & & & & & \\
\hline Salários & $\mathrm{F}$ & & & & & \\
\hline Encargos Sociais & $\mathrm{F}$ & & & & & \\
\hline Vale Transporte & $F$ & & & & & \\
\hline Alimentaçẵo & $\mathrm{F}$ & & & & & \\
\hline Assistência Médica & $\mathrm{F}$ & & & & & \\
\hline Treinamento & $F$ & & & & & \\
\hline B - CUSTOS COM FERRAMENTAS E EQUIPAMENTOS & BASEF\&E & & & & & \\
\hline Curta duracăão - reposição anual & $\bar{y}$ & & & & & \\
\hline Média duração - reposiç̧ão 2 anos & $\mathrm{V}$ & & & & & \\
\hline Longa duração - reposição 3 anos & V & & & & & \\
\hline & & & & & & \\
\hline C. CUSTOS COM VEICULOS & BASE VEICULOS & & & & & \\
\hline FIXO & $F$ & & & & & \\
\hline VARIÁVEL & V & & & & & \\
\hline & & & & & & \\
\hline D - DESPESAS ADMINISTRATIVAS E TRIBUTÁRIAS & $\mathrm{F}$ & & & & & \\
\hline & DESP. ADMIN. & & & & & \\
\hline E - ATUALIZAÇÃO DO CAPITAL DE GIRO & $\mathbf{F}$ & & & & & \\
\hline & CAP. DE GIRO & & & & & \\
\hline F - REMUNERAÇÃO DO INVESTIMENTO & $\mathrm{F}$ & & & & & \\
\hline & REM. INVEST. & & & & & \\
\hline SUBTOTAL DE CUSTOS FIXOS & $\bar{F}$ & & & & & \\
\hline SUBTOTAL DE CUSTOS VARIAVEIS & $\mathrm{V}$ & & & & & \\
\hline TOTAL GERAL & & & & & & \\
\hline
\end{tabular}

\begin{tabular}{|c|c|}
\hline QUANTIDADE DE HORAS (CAPACIDADE INSTALADA) & \\
\hline CUSTO HORA (com base na CAPACIDADE INSTALADA) & \\
\hline QUANTIDADE DE HORAS (TRABALHADAS) & \\
\hline CUSTO HORA (com base na UTILIZAÇÃO DOS RECURSOS) & \\
\hline
\end{tabular}

\begin{tabular}{|c|c|}
\hline QUANTIDADE DE HOMENS HORA (CAPACIDADE INSTALADA-7 pesSOas) & \\
\hline CUSTO HOMEM HORA (com base na CAPACIDADE INSTALADA) & \\
\hline QUANTIDADE DE HOMENS HORA (com base na UTILIZAÇÃO DOS RECURSOS) & \\
\hline CUSTO HOMEM HORA (com base na UTILIZAÇÃO DOS RECURSOS) & \\
\hline
\end{tabular}

\begin{tabular}{|l|c|}
\hline INCIDÊNCIAS SOBRE A RECEITA & \\
\hline LUCRO DESEJADO & \\
\hline PIS & \\
\hline COFINS & \\
\hline ISS & \\
\hline IMPOSTO DE RENDA + CONTRIB. SOCIAL & \\
\hline
\end{tabular}

\begin{tabular}{|c|c|}
\hline PREÇO HORA EQUIPE (com base na CAPACIDADE INSTALADA) & \\
\hline PREÇO HORA EQUIPE (com base na UTILIZAÇÃO DOS RECURSOS) & \\
\hline \multicolumn{2}{|c|}{} \\
\hline PREÇO HOMEM HORA (com base na CAPACIDADE INSTALADA) & \\
\hline PREÇO HOMEM HORA (com base na UTILIZAÇÃO DOS RECURSOS) & \\
\hline
\end{tabular}

Figura 4: Estrutura do sistema desenvolvido Fonte: os autores. 
A partir desse conjunto de variáveis, apura-se o custo e preço da hora trabalhada por equipe, comparando inclusive com o modelo de referência da ANEEL, como pode se verificar no Quadro 1 Comparativo ER/Contratada.

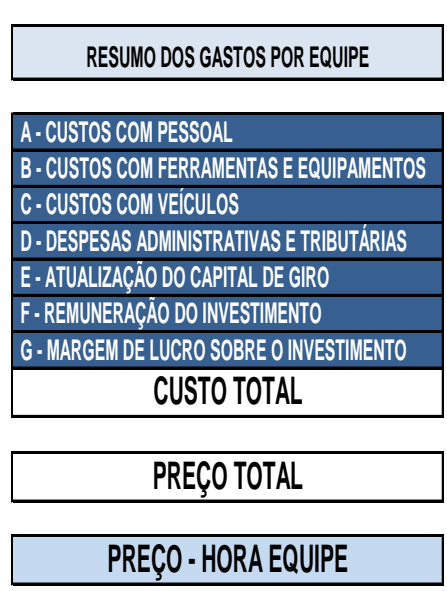

PREÇO - HORA EQUUPE
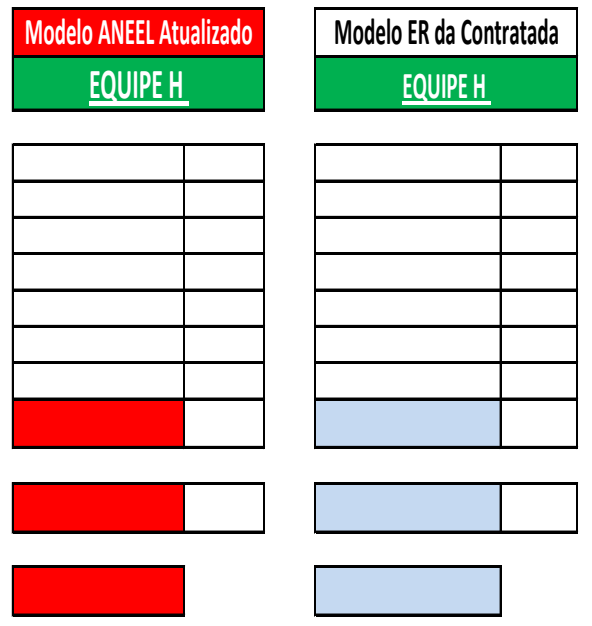
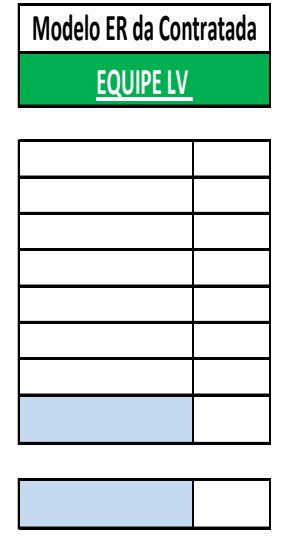

Quadro 1: Comparativo ER/Contratada Fonte: os autores.

Integrando os componentes: físico (tempos-padrões validados estatisticamente) e monetário (custo e preço hora da equipe) do modelo, calcula-se o custo e/ou o preço final de cada atividade executada pelas empresas prestadoras de serviços como, por exemplo, abrir cavas, instalar postes, etc., como pode ser visualizado no Quadro 2 de Custo das Atividades.

\section{CUSTO DAS ATIVIDADES}

\begin{tabular}{|c|c|c|c|c|c|}
\hline ATIV. & DESCRIÇÃO & LINHA & $\begin{array}{c}\text { TEMPO TOTAL } \\
\text { em minutos }\end{array}$ & $\begin{array}{c}\text { PREÇO MINUTO } \\
\text { equipe }\end{array}$ & PREÇO UNIT. \\
\hline $\mathbf{0}$ & & & & & \\
\hline 1 & & & & & \\
\hline 2 & & & & & \\
\hline 3 & & & & & \\
\hline $\mathrm{n}$ & & & & & \\
\hline
\end{tabular}

Quadro 2: Custo das Atividades

Fonte: os autores. 
Dessa forma, tem-se o modelo concluído, permitindo avaliar e simular todos os aspectos que impactam na remuneração das equipes terceirizadas, buscando uma remuneração justa.

\section{CONSIDERAÇÕES FINAIS}

A gestão estratégica requer um sistema que identifique, aprimore e evidencie alternativas para fomentar o processo decisório. A finalidade do sistema é gerar informações tempestivas que auxiliem nas escolhas que proporcionem o sucesso da organização. O (SAD) desenvolvido permite este acesso a informações e tratam de problemas pouco ou não estruturados que se convertem em soluções estruturadas, construindo assim cenários mais realistas ao momento enfrentado pelas empresas.

Nesse contexto, foi aplicado o sistema para análise e avaliação da forma de custos operacionais em Redes de Distribuição de Energia Elétrica, direcionado para a remuneração de equipes pesadas de construção e manutenção destas redes. $O$ modelo implementado na forma de SAD traduz critérios de custos que buscam aprimorar ainda mais a tarifa justa, na forma de gestão da organização estudada e dos critérios da ANNEL.

Considerou-se os custos atuais praticados, a estrutura adotada pelos prestadores de serviços, bem como se usou a referência sugerida pela ANEEL na audiência pública (AP52) referente aos módulos construtivos.

O modelo foi validado junto à empresa, considerando medições de campo, análises estatísticas para determinar os limites de confiança dos tempos das atividades, e o próprio sistema desenvolvido.

Os tempos e custos associados do modelo devem ser revisados periodicamente devido à caracterização de prestação deste tipo de serviço e sua evolução tecnológica, permitindo assim estabelecer um processo de melhoria contínua. Além dos benefícios acima citados, o trabalho permitiu: reduzir a lista de atividades através de agrupamento por semelhança, eliminação de atividades compensatórias e transparência das informações para a negociação com os prestadores de serviços. 
Por fim, sugere-se evoluir este estudo para atender as atividades de equipes leves, as quais hoje trabalham sob forma de disponibilidade, onde se pode observar potencial redução de custos de ociosidade. Uma sugestão é buscar uma lógica de estrutura de produto para aprimorar o critério de cálculo de custos neste importante setor de prestação de serviços.

\section{Agradecimento}

Os autores agradecem o apoio da concessionária de energia elétrica AES SUL Distribuidora Gaúcha de Energia SA, por meio do projeto de Pesquisa \& Desenvolvimento intitulado "Sistema de Mensuração dos Custos Operacionais em Redes de Distribuição", realizado em parceria com a Universidade do Vale do Rio dos Sinos (UNISINOS) e colaboração do PPGA/UFRGS.

\section{REFERÊNCIAS}

AKEN, J.E Van. (2005). Management Research as a Design science: Articulating the Research Products of Mode 2 Knowledge Production in Management. British Journal of Management, v.16, n.1, pp.19-36.

ATKINSON, A.; BANKER, R. KAPLAN, R.; YOUNG, S. (2000). Contabilidade Gerencial. (2 ed.). São Paulo: Atlas.

BACKER, M.; JACOBSEN, L. (1977). Contabilidade de custos: um enfoque para administração de empresas. São Paulo: McGraw-Hill.

BORNIA, A. C. e DUTRA, Marcelo. (2009). Gestão estratégica de custos invisíveis: o caso das empresas estatais transmissoras de energia elétrica do Brasil. $A B C$ Associação Brasileira de Custos, volume IV, № 2, maio/agosto.

Bookman.

. (2010). Análise Gerencial de Custos em Empresas Modernas. Porto Alegre:

GRILO, R.M. (2008). Investigação em Sistemas de Informação Organizacionais - Teses e dissertações em Portugal. Universidade de trás-os-montes e alto douro, Vila Real.

HEVNER, A.; MARCH, S. (2004). Design Science in information systems research. MIS Quarterly. Vol.28, no.1, pp. 75-105.

KEEN; P.; SCOTT-MORTON, M. (1978). Decision Support System. Reading. MA: Addison-Wesley Publishing Company. 
Sistema de informação para gestão de custos operacionais Márcio Roberto de Mello, João Luiz Becker, Daniel Porto, Adolfo Alberto Vanti

LEONE, G.S.G. (1996). Custos: planejamento, implantação e controle. (2 ed.). São Paulo: Atlas.

. (2000). Custos: um enfoque administrativo. (13 ed.). São Paulo: Atlas.

MANSON, N.J. (2006). Is operations research really research? Operations Research Society of South Africa, v.22, n.2, pp.155-180.

MARCH, S.; G. SMITH. (1995). Design and Natural Science Research on Information Technology. Decision Support Systems 15. pp. 251-266.

MARTINS, E. (2008). Contabilidade de custos. (8 ed.). São Paulo: Atlas.

MELLO, M. R. (2002). Sistema Integrado de custo-padrão, orçamento e contabilidade como instrumento de mensuração e avaliação de desempenho econômico - Estudo de caso da Gerdau Riograndense. São Leopoldo: UNISINOS. (Dissertação de Mestrado). Universidade do Vale do Rio dos Sinos, Programa de pós-graduação em Ciências Contábeis.

NAKAGAWA, M. (1995). ABC: Custeio Baseado em Atividades. São Paulo: Atlas.

PADOVEZE, C.L. (2003). Controladoria estratégica e operacional. São Paulo: Thompson.

SLOMSKI, V. (2006). Manual de contabilidade pública: um enfoque na contabilidade municipal. São Paulo: Atlas.

SOUZA, M.A.; DIEHL, C.A. (2009). Gestão de Custos: Uma abordagem integrada entre Contabilidade, engenharia e administração. São Paulo: Atlas.

SPRAGUE, R. CARLSON, E. (1982). Building Effective Decision Support Systems. NY: Prentice Hall.

SPRAGUE, R.; WATSON, H. (1989). Decision support systems: putting theory into practice. USA: Prentice-Hall.

TAKEDA, H., VEERKAMP, P., TOMIVAMA, T., YOSHIKAWAM, H. (1990). Modeling Design Cambridge, MA. Processes. Al Magazine Winter: 37-48.

TURBAN, Efrain; WETHERBE, James; McLEAN, Ephraim; LEIDNER, Dorothy E. (2010). Tecnologia da Informação para gestão: Transformando Os Negócios $\mathrm{Na}$ Economia Digital. (6 ed.). Porto Alegre: Bookman. 
Sistema de informação para gestão de custos operacionais Márcio Roberto de Mello, João Luiz Becker, Daniel Porto, Adolfo Alberto Vanti

WITTEN, I; FRANK, E. (2005). Data Mining. Practical Machine Learning Tools and Techniques. New Zealand: Morgan Kaufmann Publishers.

Data de Submissão: 06/02/2012

Data de Aceite: 12/04/2012 